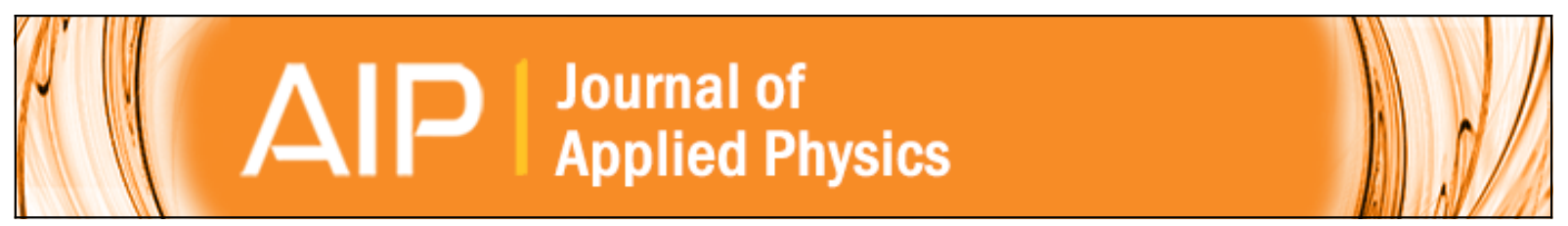

\title{
Decoupling of epitaxial graphene via gold intercalation probed by dispersive Raman spectroscopy
}

P. B. Pillai, M. DeSouza, R. Narula, S. Reich, L. Y. Wong, T. Batten, and J. Pokorny

Citation: Journal of Applied Physics 117, 183103 (2015); doi: 10.1063/1.4920931

View online: http://dx.doi.org/10.1063/1.4920931

View Table of Contents: http://scitation.aip.org/content/aip/journal/jap/117/18?ver=pdfcov

Published by the AIP Publishing

\section{Articles you may be interested in}

Structural consequences of hydrogen intercalation of epitaxial graphene on $\mathrm{SiC}(0001)$

Appl. Phys. Lett. 105, 161602 (2014); 10.1063/1.4899142

A temperature dependent measurement of the carrier velocity vs. electric field characteristic for as-grown and $\mathrm{H}$ intercalated epitaxial graphene on $\mathrm{SiC}$

J. Appl. Phys. 113, 193708 (2013); 10.1063/1.4807162

Hafnium intercalation between epitaxial graphene and $\operatorname{Ir}(111)$ substrate

Appl. Phys. Lett. 102, 093106 (2013); 10.1063/1.4793427

Raman study on the interlayer interactions and the band structure of bilayer graphene synthesized by alcohol chemical vapor deposition

Appl. Phys. Lett. 99, 151916 (2011); 10.1063/1.3651325

Probing residual strain in epitaxial graphene layers on $4 \mathrm{H}-\mathrm{SiC}\left(0001^{-}\right.$) with Raman spectroscopy

Appl. Phys. Lett. 98, 051910 (2011); 10.1063/1.3551625

Frustrated by

old technology?

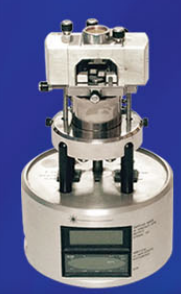

Is your AFM dead

and can't be repaired?

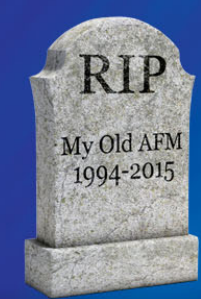

Sick of bad customer support?

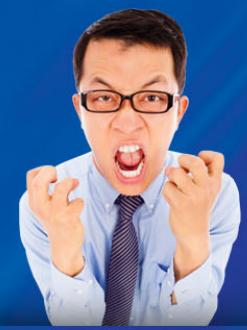

It is time to upgrade your AFM Minimum \$20,000 trade-in discount for purchases before August 31st

Asylum Research is today's technology leader in AFM

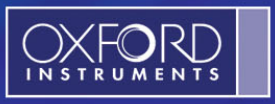




\title{
Decoupling of epitaxial graphene via gold intercalation probed by dispersive Raman spectroscopy
}

\author{
P. B. Pillai, ${ }^{1, a)}$ M. DeSouza, ${ }^{1, a)}$ R. Narula, ${ }^{2}$ S. Reich, ${ }^{2}$ L. Y. Wong, ${ }^{3}$ T. Batten, ${ }^{3}$ \\ and J. Pokorny ${ }^{4,5}$ \\ ${ }^{1}$ Semiconductor Materials and Device Group, Electronic and Electrical Engineering, University of Sheffield, \\ Mappin Street, S1 3JD Sheffield, United Kingdom \\ ${ }^{2}$ Department of Physics, Freie Universität Berlin, Arnimallee 14, 14195 Berlin, Germany \\ ${ }^{3}$ Renishaw, Old Town, Wotton-under-Edge, GL12 7DW Gloucestershire, United Kingdom \\ ${ }^{4}$ Department of Materials Science and Engineering, Sir Robert Hadfield Building, Mappin Street, \\ S1 3JD Sheffield, United Kingdom \\ ${ }^{5}$ Institute of Physics, Academy of Sciences of the Czech Republic, Na Slovance 2, 18221 Praha 8 , \\ Czech Republic
}

(Received 3 January 2015; accepted 29 April 2015; published online 12 May 2015)

\begin{abstract}
Signatures of a superlattice structure composed of a quasi periodic arrangement of atomic gold clusters below an epitaxied graphene (EG) layer are examined using dispersive Raman spectroscopy. The gold-graphene system exhibits a laser excitation energy dependant red shift of the $2 \mathrm{D}$ mode as compared to pristine epitaxial graphene. The phonon dispersions in both the systems are mapped using the experimentally observed Raman signatures and a third-nearest neighbour tight binding electronic band structure model. Our results reveal that the observed excitation dependent Raman red shift in gold EG primarily arise from the modifications of the phonon dispersion in gold-graphene and shows that the extent of decoupling of graphene from the underlying $\mathrm{SiC}$ substrate can be monitored from the dispersive nature of the Raman 2D modes. The intercalated gold atoms restore the phonon band structure of epitaxial graphene towards free standing graphene. (C) 2015 AIP Publishing LLC. [http://dx.doi.org/10.1063/1.4920931]
\end{abstract}

\section{INTRODUCTION}

Graphene is a deceptively simple two-dimensional arrangement of carbon atoms, which is the building material of other graphitic forms of carbon. ${ }^{1}$ Its superior electronic properties make it a promising candidate for future electronic applications. ${ }^{2-4}$ However, the finite transmission probability of electrons in graphene across potential barriers regardless of their height and width, called Klein tunnelling, ${ }^{5}$ poses a serious challenge in the applicability of this material to digital electronics and photonics. This can be overcome by inducing a controllable band gap in the material. However, limitations in resolution of resist based electron beam lithography of sub nanometre dimensions to induce such a band-gap by quantum confinement poses significant technical challenges. ${ }^{6}$ On the other hand, the concept of selfassembled superlattice structures offers a more realistic option to realise electronic applications without complex lithography. ${ }^{7}$ Large scale graphene superlattices have recently been demonstrated by intercalating metal atoms below epitaxial graphene (EG) or by the growth of graphene on lattice mismatched metal substrates. ${ }^{8-10}$ Recently, graphene/boron-nitride heterostructures have emerged as a very promising system for band engineering of graphene. ${ }^{11}$ Theoretical investigations by Park et al. ${ }^{12}$ revealed that the application of a periodic potential by suitable patterns results in highly anisotropic charge carrier behaviour in graphene. An experimental evidence for this was reported by

\footnotetext{
${ }^{\text {a) }}$ Authors to whom correspondence should be addressed. Electronic addresses: p.pillai@sheffield.ac.uk and m.desouza@sheffield.ac.uk.
}

Yankowitz et al. ${ }^{10}$ They observed a rotation dependent Moiré pattern of graphene on hexagonal boron nitride (h-BN), which acts as a periodic potential on the graphene lattice, leading to the generation of new Dirac points in the electronic spectrum and anisotropic charge carrier velocity. ${ }^{10}$ Similar to their observation, we have earlier reported a superlattice structure of gold atoms below epitaxial graphene which exhibits a unique standing wave pattern in its charge density. ${ }^{8,13}$ These intercalated atomic clusters locally screen the charge transfer from the substrate to graphene leading to charge anisotropy in graphene.

Epitaxial graphene growth on the $\mathrm{Si}$ terminated $\mathrm{SiC}$ surface results initially in a carbon rich buffer layer in which every third carbon atom is covalently bonded to the underlying $\mathrm{Si}$ atoms. ${ }^{14}$ The proximity of the buffer layer below graphene imparts strong n-type doping and strain on the subsequently grown monolayer graphene. Intercalating metal atoms between the buffer layer and monolayer graphene has been suggested as a suitable method to decouple the graphene and to realize the formation of quasi free-standing graphene on $\mathrm{SiC} .{ }^{13,15}$ In the present study, it is shown via Raman spectroscopy that the decoupling of graphene by intercalated gold atoms can be understood by considering the dispersive nature of the 2D modes. An excitation dependent Raman red-shift observed in gold-intercalated epitaxial graphene in comparison to pristine epitaxial graphene is investigated by considering the contributions from strain, doping, and phonon dispersion in graphene due to intercalated gold atoms. Here, the term "pristine EG" is used to address epitaxial graphene prior to the deposition of gold and gold-EG 
for gold intercalated EG. Spectroscopic features acquired at different incident laser polarizations and dispersive micro Raman measurements reveal that the origin of the excitation dependent Raman red-shift observed in the case of goldintercalated epitaxial graphene can only be explained on the basis of modification of their phonon band structure.

\section{EXPERIMENT AND THEORY}

Graphene samples were prepared in UHV by the annealing of $\mathrm{n}$-doped $\mathrm{SiC}(0001)$ at $900 \mathrm{~K}$ for several hours and subsequent annealing at $1500 \mathrm{~K}$ for $30 \mathrm{~min}$. The deposition of gold on graphene was carried out at room temperature using a homemade Knudsen cell calibrated using a quartz crystal microbalance. The sample was further annealed at $1000 \mathrm{~K}$ for $5 \mathrm{~min}$. The intercalated areas of gold grow in size up to several micrometers in subsequent annealing cycles. ${ }^{8,13}$ Raman spectra are recorded using a Renishaw inVia micro Raman spectrometer working in back scattering geometry using excitation wavelengths 514, 532, 633, and $785 \mathrm{~nm}$. A laser power of $3 \mathrm{~mW}$ is used with an objective of $100 \times$ magnification and numerical aperture of 0.85 . The sample surface topography was recorded using an Omicron-Low Temperature Scanning Tunneling Microscope (LTSTM) working at $77 \mathrm{~K}^{13}$ The electronic band structure in pristine and gold-EG are deduced by fitting their experimentally reported Angleresolved photoemission spectroscopy (ARPES) band disper$\operatorname{sion}^{16}$ using a third nearest neighbor tight binding (3NN-TB) model [next nano $3^{17}$ ]. The analytical expressions used in the model are previously described by Reich et al. ${ }^{18}$

\section{RESULTS AND DISCUSSION}

Fig. 1(a) shows a $5 \times 5 \mathrm{~nm}^{2}$ STM image of gold intercalated graphene where the clusters appear as bright protrusions (image processing was carried out using WSxM. ${ }^{19}$ ). The honeycomb structure is apparent on top of the clusters, which implies that the clusters are intercalated below the monolayer graphene. The inset of Fig. 1 (a) is a $10 \times 10 \mathrm{~nm}^{2}$ image of the irregular distribution of clusters captured at a higher bias voltage. The bottom of Fig. 1(a) represents a schematic model which indicates the position of the gold clusters $(\mathrm{Au})$ as lying between the buffer layer (BL) and the first layer of graphene (MG). ${ }^{13}$ Fig. 1(b) shows the experimental Raman peaks corresponding to pristine EG at a laser excitation of $2.41 \mathrm{eV}$. The resultant spectrum after subtracting the contribution from the underlying $\mathrm{SiC}$ substrate is shown as the curve at the top. A direct observation of the $\mathrm{G}$ band in EG is obscured by the strong modes emanating from the $\mathrm{SiC}$ substrate (between wavenumber $1400-2000 \mathrm{~cm}^{-1}$ ). Hence, in the present study, only the 2D Raman modes are considered. The number of graphene layers is also confirmed from Raman spectra (see supplementary material, Fig. S1). The excitation dependence of the 2D mode in the gold intercalated epitaxial graphene (red curves) is shown in Fig. 1(c). The black curves at the bottom denote the reference pristine epitaxied graphene with vertical lines indexing their maximum values. Care was taken to ensure Raman measurements were collected from the same location on each sample. Fig. 1(d) shows the frequency dispersion of the $2 \mathrm{D}$ mode with laser energy in pristine and gold EG samples. Fig. 1(e) shows the Raman spectral map of the 2D peak position of goldintercalated graphene. Fig. 1(f) represents the Raman spectral map for pristine EG obtained from a line scan data over the range shown in the figure as a reference.

A closer look into the double resonant 2D Raman modes of pristine EG and gold intercalated EG shown in Figs. 1(c) and 1(d) reveals (a) an intriguing excitation dependent Raman shift between the two (b) the gold intercalated EG exhibits a steeper 2D dispersion as compared to the pristine EG. The experimental value of the red shift changes from approximately $3 \mathrm{~cm}^{-1}$ to $17 \mathrm{~cm}^{-1}$ as the excitation energy changes from $2.41 \mathrm{eV}$ to $1.58 \mathrm{eV}$. The error bar in Fig. 1(d) corresponds to the uncertainty in the Lorentzian peak maximum for the 2D mode. From the laser energy dependence, a 2D peak dispersion value of $102 \mathrm{~cm}^{-1} / \mathrm{eV}$ and $124 \mathrm{~cm}^{-1} / \mathrm{eV}$ is deduced for pristine and gold-EG, respectively. The origin of the first and second order double resonant Raman mode in graphene is well understood in the literature. ${ }^{20-24}$ It has been identified that the phonon mode involved in the origin of the 2D mode in graphene is the transverse optical (TO) near the $\mathrm{K}$ point of the Brillouin zone. ${ }^{20}$ As the excitation laser energy increases, the incident photon excites electronic levels farther from the $\mathrm{K}$ point and results in progressively higher phonon momenta and correspondingly higher energies. This results in the energy dispersive behaviour of the 2D Raman mode in graphene. ${ }^{20}$ The three possible origins for the observed variations in the Raman spectra between the pristine and gold EG are (a) strain generated in gold-EG, (b) doping in gold-EG, and (c) change in phonon dispersion in gold-EG due to intercalated gold atoms. In Secs. III A-III C, the dependence of each of these factors on the observed Raman behaviour reported in Figs. 1(c) and 1(d) are discussed.

\section{A. Polarization dependent Raman spectroscopy and role of strain}

Strain in graphene significantly alters the Raman modes through a modification of the electronic and phonon band structure. ${ }^{25-27}$ The influence of strain on the line shape and frequency of the 2D Raman mode of graphene has been well understood in the literature. ${ }^{25,28-30}$ Strain splits the 2D peak in graphene into two components with the magnitude of split depending on the direction of the strain in graphene. ${ }^{29,30}$ The component of the $2 \mathrm{D}$ peak with a smaller frequency is represented as $2 \mathrm{D}^{-}$and that with higher frequency as $2 \mathrm{D}^{+}$. In the case of strained graphene, when the incident laser polarisation is rotated with respect to the strain direction, the spectral weight of the $2 \mathrm{D}$ mode shifts from the $2 \mathrm{D}^{-}$component to the $2 \mathrm{D}^{+}$component. That is, when the polarisation of the incident laser is parallel to the strain direction, the $2 \mathrm{D}^{-}$component is dominant and when perpendicular, $2 \mathrm{D}^{+}$component becomes dominant. The separation between the two components depends on the magnitude of strain. ${ }^{29,30}$

To evaluate the strain present in pristine EG and goldEG, we have conducted polarisation dependent Raman spectroscopy on these samples by using an excitation wavelength 

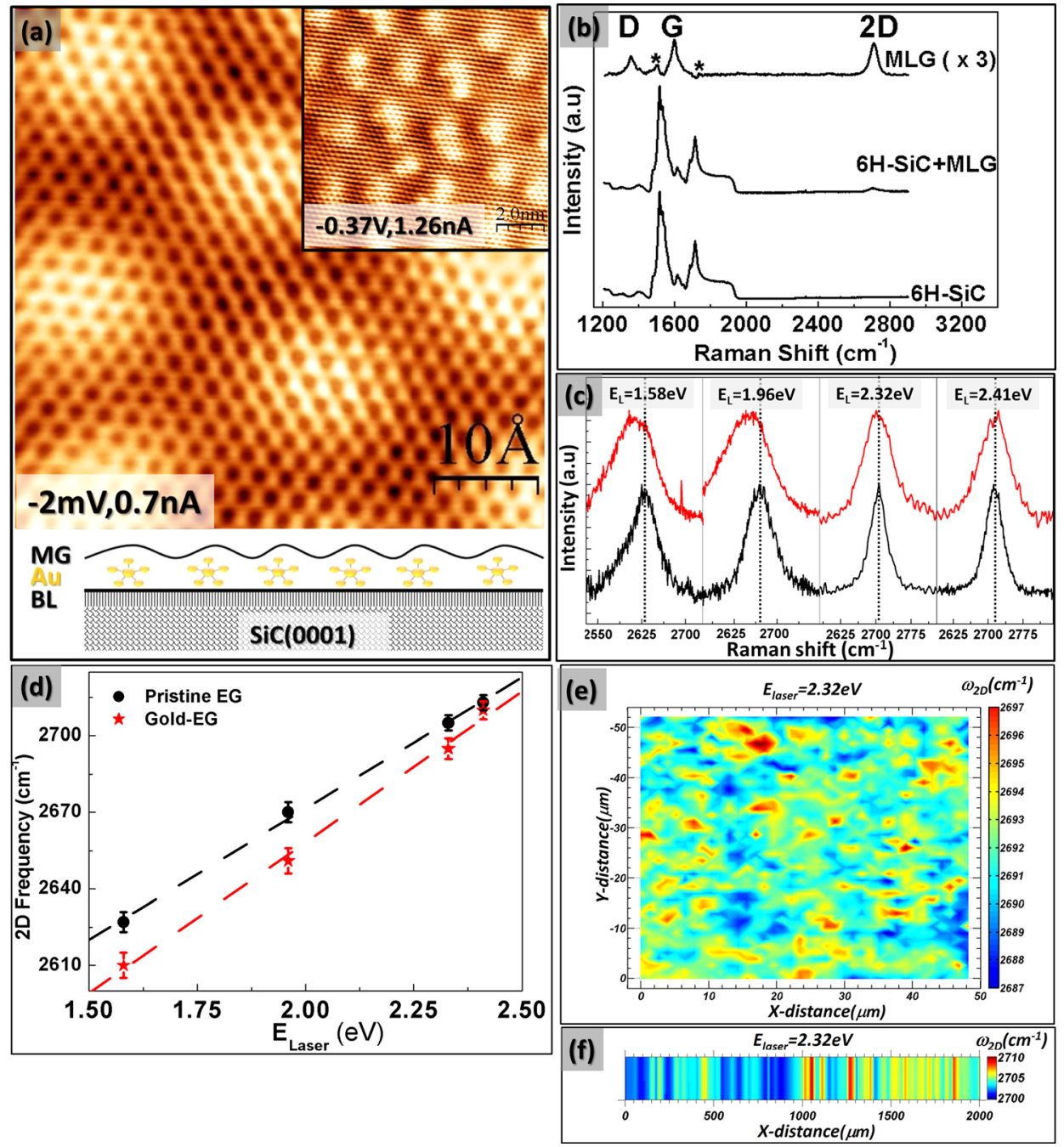

FIG. 1. (a) Topographic atomic resolution STM images showing monolayer epitaxial graphene with gold atomic clusters intercalated below. Figure inset is an STM image captured at higher bias $(-0.37 \mathrm{~V})$, which shows the distribution of clusters below the graphene. The honeycomb structure of graphene is clearly visible on top of these clusters. The bottom part of Fig. 1(a) shows a schematic model revealing the position of gold clusters as intercalated between the buffer layer and the first layer of graphene. (b) Raman spectrum collected from (i) the SiC substrate (bottom curve), (ii) monolayer epitaxial graphene grown on SiC substrate (middle curve), and (iii) the resultant spectrum obtained by subtracting the contribution from the substrate (top curve). Non-subtracted substrate features are indicated by a "**" in the top curve. The positions of respective Raman modes are indexed as D, G, and 2D which are the defect induced D band, first order-Raman G mode and double resonant two phonon-2D modes, respectively. (c) A comparison of pristine and gold intercalated EG 2D modes at various laser excitation energies. The laser energy corresponding to each curve is indexed, and vertical dotted lines indicate the maximum in pristine epitaxial graphene. (d) The 2D mode spectral dispersions in pristine (circles) and gold intercalated graphene (star) are shown together to reveal an excitation dependent red shift between the two (e) and (f) Raman spectral map of the position of the 2D peak in gold-intercalated graphene and pristine EG, respectively, collected using a laser energy of $2.32 \mathrm{eV}$.

of $532 \mathrm{~nm}$ and a half wave plate is introduced in the incident laser path to rotate the polarisation. The relative angle between the incident and analyser polarisation $(\beta)$ is varied in steps of $20^{\circ}$ and the $2 \mathrm{D}$ mode is acquired using identical experimental conditions in all cases. Fig. 2(a) shows the evolution of the 2D peak in pristine EG when $\beta$ is varied from nearly parallel $\left(190^{\circ}\right)$ to crossed polarizations $\left(90^{\circ}\right.$ or $\left.270^{\circ}\right)$ (top to bottom). The normalized intensity of the 2D mode in pristine EG is plotted with the relative angle $\beta$ as shown in Fig. 2(b). Yoon et al. ${ }^{31}$ in their report revealed a relation connecting the intensity of the 2D Raman mode and the relative angle between the incident and analyser polarisation $\beta$ as

$$
I_{\beta}=\frac{I_{\|}}{3}\left(2 \cos ^{2} \beta+1\right)
$$

where $I_{\|}$is the intensity of the $2 \mathrm{D}$ is peak when incident and scattered polarizations are parallel to each other. As expected in the case of exfoliated graphene, the observed maximum intensity in pristine EG when the incident and analyser polarisations are parallel is $\approx 3$ times the minimum intensity recorded when the two polarisations are perpendicular to each other. The 2D peak shows no clear splitting or anisotropy when the incident polarisation is rotated from the parallel to the perpendicular directions and it can be fitted with a single Lorentzian of width $\sim 40 \mathrm{~cm}^{-1}$ (not shown). This value is different from that of exfoliated graphene $e^{29,30}$ $\left(\sim 32 \mathrm{~cm}^{-1}\right)$ and the difference can be attributed to the $\mathrm{n}$-doping and strong interaction of graphene with the underlying $\mathrm{SiC}$ substrate. However, in the case of gold-EG, when the incident polarization is rotated from the perpendicular to 

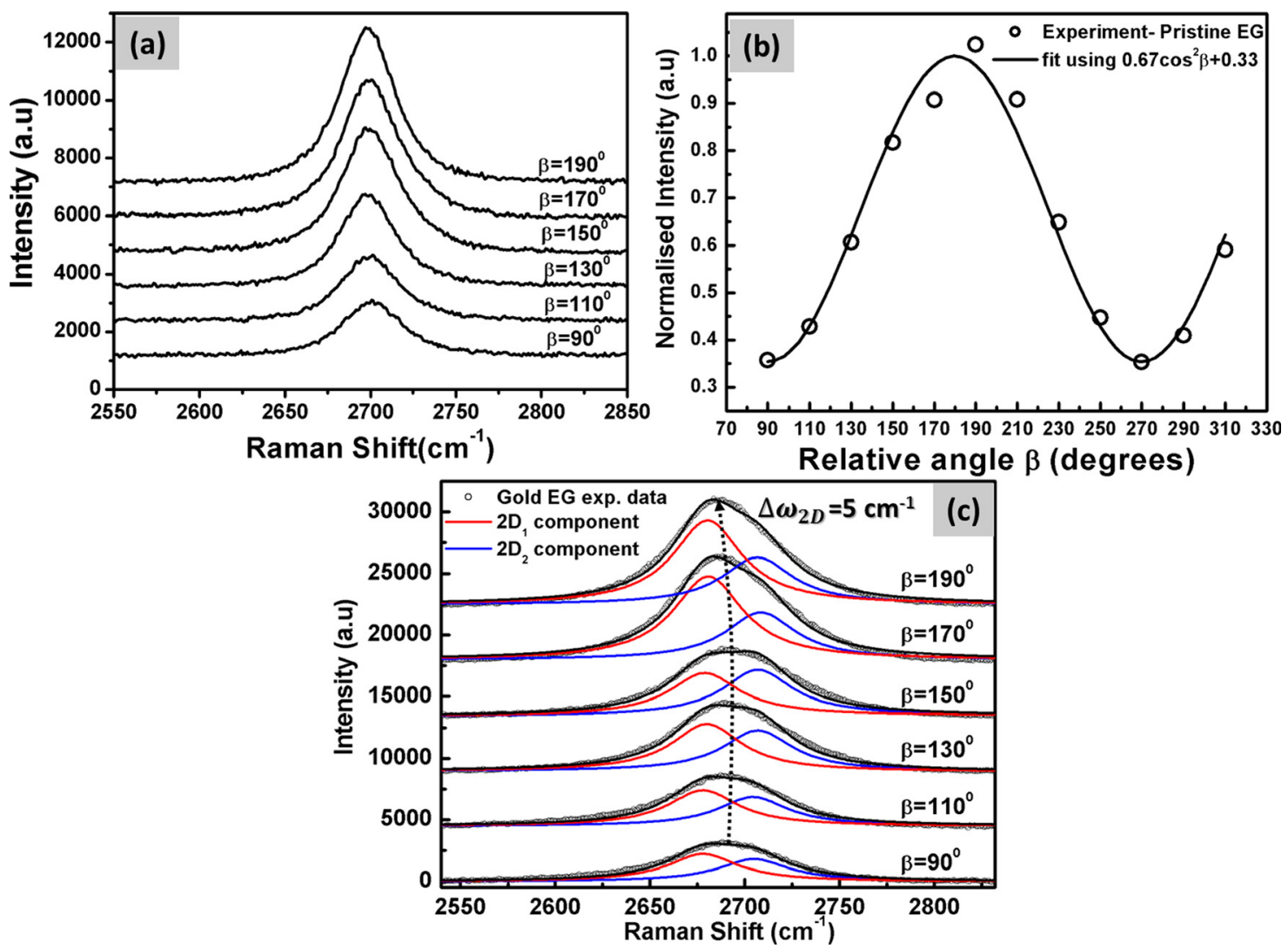

FIG. 2. (a) Evolution of the 2D Raman mode in pristine EG when incident laser polarization is rotated from nearly parallel $\left(\beta \approx 190^{\circ}\right)$ to crossed polarizations $\left(\beta \approx 90^{\circ}\right)$ (top to bottom) where $\beta$ is the relative angle between the incident and analyzer polarization. (b) Normalized intensity of the $2 \mathrm{D}$ Raman mode in pristine EG and its fit using the equation $I_{\beta}=0.67 \cos ^{2} \beta+0.33$. The spectral shape and position shows no dependence on $\beta$ and reveals strain is negligible or absent in pristine EG. (c) Gold-EG exhibits a marginal shift in the 2D frequency $\left(5 \mathrm{~cm}^{-1}\right)$ when the polarization of the incident laser is rotated from the parallel to perpendicular directions. The $2 \mathrm{D}$ mode in gold-EG at each polarization condition is resolved into two Lorentzian components represented as $2 \mathrm{D}_{1}$ (red curves) and $2 \mathrm{D}_{2}$ (blue curves). The spectra in Figs. 2(a) and 2(c) are vertically shifted for clarity.

the parallel directions, the spectral weight of the $2 \mathrm{D}$ peak is red-shifted by $\approx 5 \mathrm{~cm}^{-1}$.

A more detailed analysis by resolving the observed 2D peak into its constituent components is required to draw a stronger conclusion. The 2D peak shows a considerable broadening with full width half maximum (FWHM) of the peak of $\sim 70 \mathrm{~cm}^{-1}$. The resultant 2D peak under various polarisation conditions in gold-EG is fitted using two Lorentzian components as shown in Fig. 2(c). In the case of strained exfoliated graphene, it has been previously observed that the $2 \mathrm{D}^{-}$peak is linearly polarised (i.e., the intensity is maximum when the incident polarisation is parallel to the strain axis) and the $2 \mathrm{D}^{+}$peak is perpendicularly polarised (i.e., the intensity is maximum when the incident polarisation is perpendicular to the strain axis). ${ }^{29,30,32}$ In the present experiment, in the case of gold-EG, the observed 2D peak can be resolved into two components, a $2 \mathrm{D}_{1}$ component at the lower energy or wavenumber, analogous to the $2 \mathrm{D}^{-}$ component in strained exfoliated graphene, and $2 \mathrm{D}_{2}$ component, which is the component with the higher wavenumber. Under a constant strain in graphene, as the incident laser polarization is rotated with respect to the direction of strain, only the relative intensity between the split components changes, but their position remains the same. ${ }^{29}$ In our case, the difference of frequency between the split components $2 \mathrm{D}_{1}$ and $2 \mathrm{D}_{2}$ is $\approx 28 \mathrm{~cm}^{-1}$ corresponding to a strain level of
$0.8 \%$ in exfoliated graphene according to Ref. 30. However, as compared to exfoliated graphene, our gold-EG samples show a 2D frequency difference $\left(\omega_{2 D}\right.$ (exfoliated $)$ $-\omega_{2 D}($ gold $-E G)$, where $\omega_{2 D}$ is the position of $2 \mathrm{D}$ peak without resolving into the two components $2 \mathrm{D}_{1}$ and $2 \mathrm{D}_{2}$ ) of only $\sim 10-20 \mathrm{~cm}^{-1}$ whereas Raman shift corresponding to a $0.8 \%$ strain in graphene is $\sim 51 \mathrm{~cm}^{-1}$ (for uniaxial strain, Ref. 32). Hence, the possibility of such high levels of strain in our samples can be discarded. In the case of 2D modes in graphene, it is known that assuming an isotropic phonon dispersion results in a nearly identical frequency for phonons along $K-\Gamma$ and $K-\mathrm{M}$ directions of the Brillouin zone. ${ }^{33}$ Recent polarization dependent Raman measurements revealed that an imperfect compensation of the trigonal warping of electron and phonon, results in a strong contribution to the broadening of the 2D Raman feature by the phonon modes away from the lines of high-symmetry. ${ }^{34}$ In such a case, the 2D mode can be resolved into two components arising from the asymmetric contributions of inner and outer phonons and the separation between the split components $\left(2 \mathrm{D}_{1}\right.$ and $\left.2 \mathrm{D}_{2}\right)$ is highly dependent on the charge density in graphene. ${ }^{34}$ This suggests that the observed $2 \mathrm{D}_{1}$ and $2 \mathrm{D}_{2}$ components in the case of gold-EG cannot be fully attributed to the strain induced splitting of 2D modes. Moreover, depolarised Raman spectra at $\mathrm{E}_{\text {Laser }}=2.32 \mathrm{eV}$ (Fig. 3) reveals that gold intercalation results in a change of the position of 

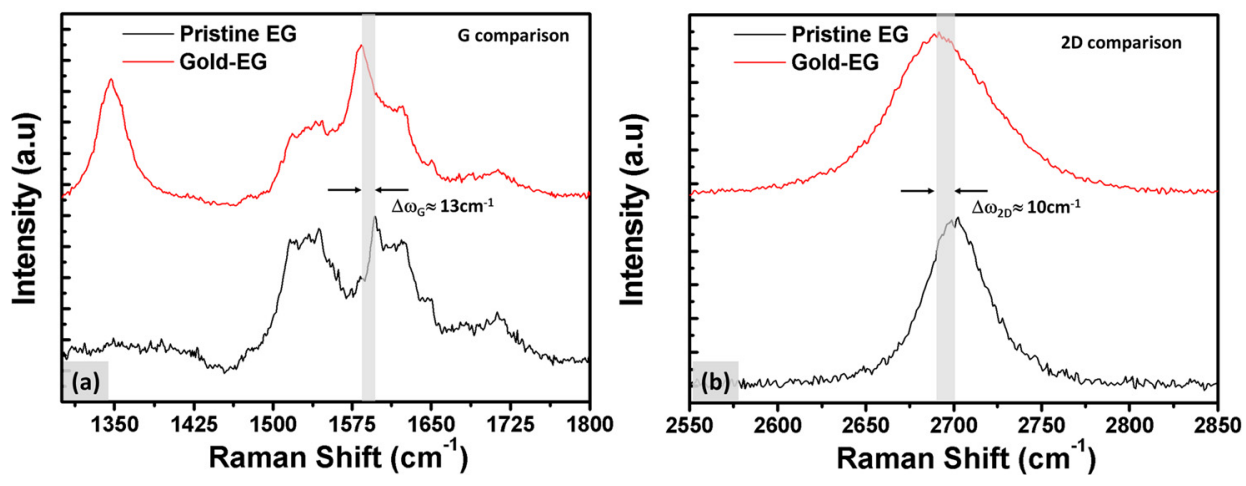

FIG. 3. (a) Depolarised Raman spectra of $\mathrm{G}$ (a) and 2D (b) peak regions of pristine and gold-EG using a laser energy of $2.33 \mathrm{eV}$. The gold-EG shows a $G$ and $2 D$ peak shift of $\sim 13 \mathrm{~cm}^{-1}$ and $10 \mathrm{~cm}^{-1}$, respectively, in comparison to its pristine counterpart. The gold-EG spectra in Figs. 3(a) and 3(b) are vertically shifted for clarity.
$\mathrm{G}$ and $2 \mathrm{D}$ modes in $\mathrm{EG}$ such that the ratio of the difference in frequencies of the modes, $\Delta \omega_{2 \mathrm{D}} / \Delta \omega_{\mathrm{G}} \approx 0.77$, is significantly different from strain induced changes in the Raman modes where a ratio of 2.5 or more may be expected. ${ }^{35}$

\section{B. Role of doping}

It has been shown in Fig. 1(d) that the gold-EG exhibits a steeper 2D mode dispersion as compared to pristine EG. Following the report by Attaccalite et al., ${ }^{36}$ electron or hole doping may reduce the $\mathrm{D}$ or $2 \mathrm{D}$ peak dispersion value in graphene by changing the dispersion of the TO phonon. The higher value of the $2 \mathrm{D}$ mode dispersion in gold-EG can be attributed to a reduction in the intrinsic n-doping present in the sample. However, according to Das et al., ${ }^{37}$ the 2D mode in graphene shows less sensitivity to a variation of electron doping in the range $0-2 \times 10^{13} \mathrm{~cm}^{-2}$ and p-doping results in unique blue shifted $\mathrm{G}$ and $2 \mathrm{D}$ modes contrary to the red shifted peaks in the present experiment. Consistent with this observation, Berciaud et al. ${ }^{34}$ also reported an unintentional p-doping of graphene on a $\mathrm{Si} / \mathrm{SiO}_{2}$ substrate, which results in a blue shifted 2D peak with symmetrical broadening. To envisage the doping variations in pristine and gold EG samples in the observed Raman shift rates, the carrier concentration in graphene is evaluated as $n\left(E_{F}\right)=\frac{1}{\pi}\left[\frac{E_{F}}{\hbar v_{F}}\right]^{2}$, where $\mathrm{E}_{\mathrm{F}}$ is the Fermi energy measured from the Dirac point. It has been observed experimentally from ARPES that the Fermi level is $\sim 0.29 \mathrm{eV}$ and $\sim 0.23 \mathrm{eV}$ away from the Dirac point in pristine and gold-EG, respectively. ${ }^{16}$ (The samples studied in ARPES and in the present study are prepared in the same set up). However, there may be sample to sample variation in doping level even grown in same furnace. To minimize this variation, samples with identical properties as reported in Ref. 16 are chosen in this study (see supplementary material, Fig. S2). This implies an electron density of $5.4 \times 10^{12} \mathrm{~cm}^{-2}$ (using $v_{F}=1 \times 10^{6} \mathrm{~m} / \mathrm{s}$ ) and $2.74 \times 10^{12} \mathrm{~cm}^{-2}$ (using $v_{F}=1.2 \times 10^{6} \mathrm{~m} / \mathrm{s}$ ) in pristine and gold-EG, respectively. This implies a marginal reduction of the intrinsic n-doping in epitaxial graphene as depicted above and cannot be considered as the observed red shift of 2D mode at the investigated laser energies.

\section{Electron and phonon dispersion in graphene}

As discussed earlier, the 2D modes exhibit a significant broadening in the case of gold-EG as compared to pristine
EG. In the case of isotropic electron and phonon dispersion, the FWHM of the 2D mode is derived as ${ }^{33}$

$$
\Gamma_{2 D}=4 \sqrt{2^{2 / 3}-1} \frac{v_{\text {phonon }}}{v_{F}} \gamma_{e h}
$$

where $v_{\text {phonon }}\left(v_{F}\right)$ is the phonon (Fermi) velocity and $\gamma_{e h}$ is the broadening parameter empirically derived by Venezuela et al., ${ }^{38}$ for laser energy $E_{\text {Laser }} \geq 1 \mathrm{eV}$ as

$$
\gamma_{\text {eh }}=\left(18.88\left|E_{\text {Laser }}\right|+6.802 E_{\text {Laser }}^{2}\right) \mathrm{meV} \text {. }
$$

Hence, the theoretically expected dependence of $\Gamma_{2 D}$ is such that it decreases with laser energy $\left(\mathrm{E}_{\text {Laser }}\right)$ as the reduction in $\gamma_{e h}$ due to decrease in $\mathrm{E}_{\text {Laser }}$ predominates over the change of $\frac{v_{\text {phonon }}}{v_{F}}$. However, similar to the case of suspended graphene, ${ }^{34}$ the present experimental data also show a noteworthy increase of $\Gamma_{2 D}$ as laser energy decreases (Fig. 1(c)). It has been shown earlier in graphene that the effects of trigonal warping of electrons and phonons are opposite ${ }^{39}$ and cancel out, resulting in a symmetric $2 \mathrm{D}$ mode with broadening, following Eq. (2). However, in the current case, the effects of trigonal warping may not cancel each other, which results in the broadening of the $2 \mathrm{D}$ mode in gold $\mathrm{EG}$. Moreover, considering the rate of shift of $\omega_{2 D}$ with respect to the photon frequency $\left(\omega_{\text {Laser }}\right)$ calculated from the observed 2D mode dispersion as $^{29}$

$$
\frac{\partial \omega_{2 D}}{\partial \omega_{\text {Laser }}}=2 \frac{\nu_{\text {phonon }}}{v_{F}} .
$$

Using the $v_{F}$ evaluated from ARPES for these samples, ${ }^{16}$ the ratios of TO phonon and Fermi velocities in pristine and gold EG are evaluated as $6.36 \times 10^{-3}$ and $7.745 \times 10^{-3}$, respectively. These values depict approximately $22 \%$ increase in the slope of the phonon dispersion in gold-EG in comparison to pristine epitaxial graphene.

Poncharal et al. established that similar excitation dependent Raman shift in 2D modes occurs because of phonon band structure modifications in folded graphene. ${ }^{39}$ A similar excitation dependence was also reported due to strain induced modifications of phonon and electron dispersion in graphene [supplementary material of Ref. 29]. Therefore, in this work, phonon dispersion as a probable cause of Raman redshift in gold-EG is evaluated using the 3NN TB-band structure combined with the experimentally observed Raman modes at various laser energies. The $3 \mathrm{NN}$ TB parameters have been fitted to the experimental 
TABLE I. TB parameters used in the model to fit the experimental valence band dispersion for pristine and gold-EG reported in Ref. 16.

\begin{tabular}{lccccccc}
\hline \hline Sample & $\varepsilon_{2 p}$ & $\gamma_{0}(\mathrm{eV})$ & $s_{0}$ & $\gamma_{1}(\mathrm{eV})$ & $s_{1}$ & $\gamma_{2}(\mathrm{eV})$ & $s_{2}$ \\
\hline Pristine-EG & -0.58 & -2.8 & 0.1 & -0.08 & -0.06 & -0.275 & 0.003 \\
Gold-EG & -0.59 & -3.31 & 0.03 & -0.1 & -0.08 & -0.35 & -0.006 \\
\hline \hline
\end{tabular}

valence band dispersion observed by ARPES, ${ }^{16}$ as shown in Table I.

Fig. 4(a) shows the two dimensional theoretical electronic structure obtained from the above approach. A weak p-doping is present in gold intercalated EG, which corresponds to an approximate energy shift of $60 \mathrm{meV}$ from the Dirac energy of pristine-EG. ${ }^{16}$ The gold-EG exhibits a steeper electronic dispersion compared to its pristine counterpart which could arise either due to the reduced interaction of graphene with the substrate or decoupling from the buffer layer. In Fig. 4(b), the triangular contour around the K point (represented by a red "+" symbol) shows the electronic energy contour corresponding to an experimentally used laser excitation of $1.58 \mathrm{eV}$. (This electronic equi-energy contour is obtained by considering the valence and conduction bands separated by $1.58 \mathrm{eV}$ symmetrically across the Dirac energy.) Similarly, the contour around the $\mathrm{K}^{\prime}$ point corresponds to valence and conduction band sections separated by an energy value of $\mathrm{E}_{\mathrm{Laser}}-2 * \omega_{\text {phonon, which repre- }}$ sents the de-excited state obtained by the emission of a light quanta of energy $\mathrm{E}_{\text {Laser }}-2 * \omega_{\text {phonon. }}$. Here, $\omega_{\text {phonon }}$ is the energy of the $2 \mathrm{D}$ peak observed experimentally for a laser excitation of $\mathrm{E}_{\mathrm{Laser}}=1.58 \mathrm{eV}$. All possible phonon vectors $\mathrm{q}_{\mathrm{i}}$ corresponding to this $2 \mathrm{D}$ phonon transition are determined by translating the contour around $\mathrm{K}$, to an equivalent tangential position on the contour around $\mathrm{K}^{\prime}$, as illustrated by the dotted arrow in Fig. 4(b). ${ }^{38}$ The evaluated phonon iso-energy contours relevant to the experimentally used laser excitations (1.58-2.41 eV) for pristine EG (circles) and gold EG (lines) are shown in Fig. 4(c). Please note that these phonon contours are derived from the $3 \mathrm{NN}$ TB electronic dispersion and hence the number of data points corresponds to the data points on the electronic contours from the TB theoretical dispersion. Fig. 4(d) shows the interpolated 3D surface of the TO phonon branch in pristine EG, constructed from the phonon iso-energy contours corresponding to the observed 2D mode energies at the various laser excitations using the mesh grid function in MATLAB. Fig. 4(e) shows the calculated TO phonon dispersion in pristine EG (black) and gold-EG (red), respectively, along the K-M direction of the Brillouin zone where $\mathrm{q}=0$ corresponds to the $\mathrm{K}$ point. The symbols in Fig. 4(e) represent the experimentally measured Raman 2D
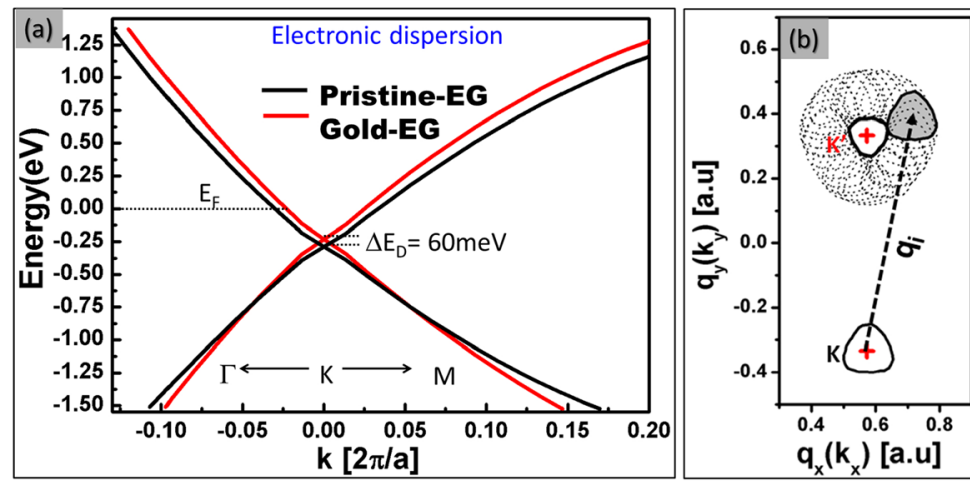
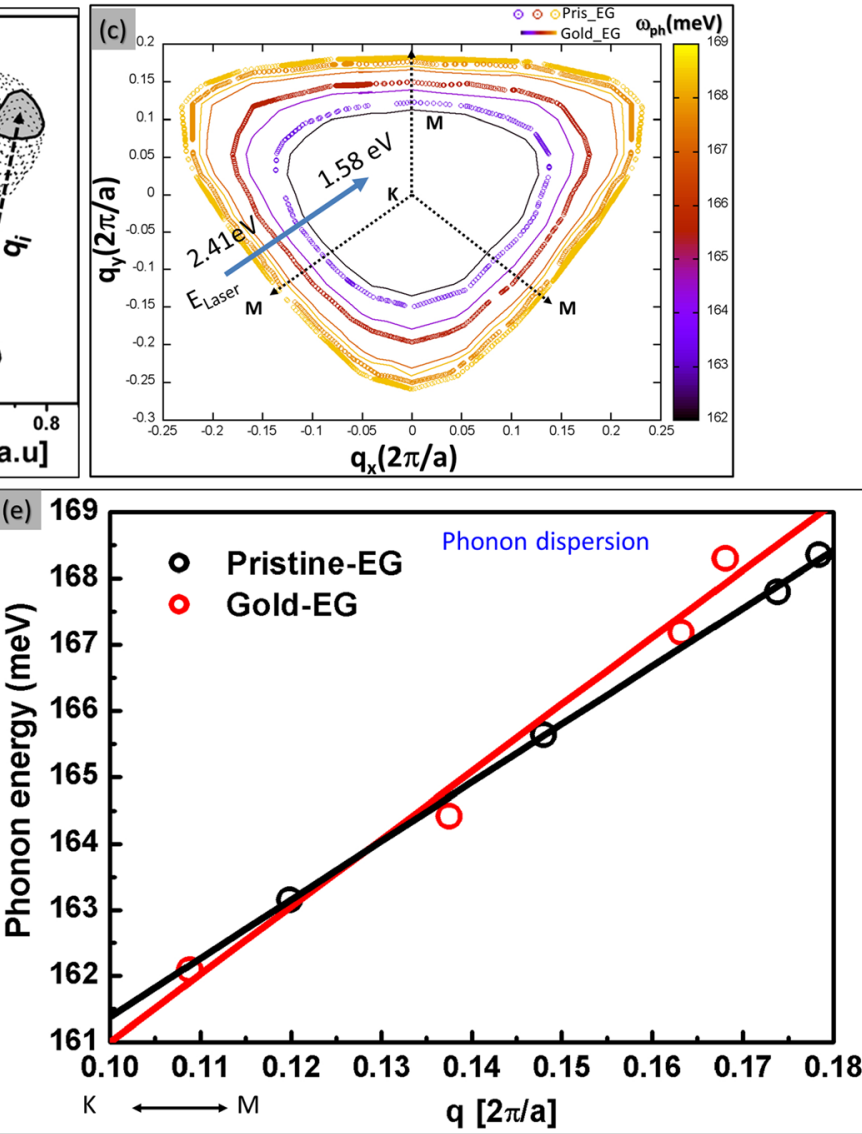

FIG. 4. (a) Third nearest neighbor tight binding (3NN-TB) band structure along the $\Gamma-\mathrm{K}-\mathrm{M}$ direction calculated by fitting the ARPES valence band structure of pristine and gold intercalated graphene reported in Ref. 16. (b) An Illustration of construction of all possible intervalley phonon momenta of transitions corresponding to the 2D mode at an experimentally used laser energy of $1.58 \mathrm{eV}$. (c) Phonon equi-energy contours relevant to the experimentally used laser excitations (1.58-2.41 eV) for pristine EG (circles) and gold EG (lines). (d) Interpolated 3D surface of the TO phonon branch in pristine EG, constructed from the phonon iso-energy contours corresponding to the observed 2D mode energies at the various laser excitations. (e) The calculated phonon dispersion and fit of the data along the K-M direction of the Brillouin zone in the case of pristine and gold EG. 
mode energies plotted against the calculated q vectors. The lines are fit to the symbols using the expression

$$
\omega(q)=\omega_{k}+\frac{\lambda_{k}}{4}\left(v_{F}^{2} q^{2}-\omega_{k}^{2}\right)^{1 / 2},
$$

where $\lambda_{k}$ is the dimensionless electron phonon coupling (EPC), $v_{F}$ is the Fermi velocity and $\omega_{k}$ is the energy of the TO phonon at the K point ${ }^{40}$ which yields $\lambda_{k}=0.056 \pm 0.001$ for pristine EG (where $\nu_{F}=6.2 \mathrm{eVA}$ has been used obtained as the slope of the electronic bands from ARPES ${ }^{16}$ ) and for gold-EG a value for $\lambda_{k}=0.054 \pm 0.001$ (using $v_{F}$ $=7.5 \mathrm{eV} \AA$ ) is obtained. The iTO phonon dispersion is steeper in the case of gold-EG and the slope of the phonon dispersions along the K-M direction is qualitatively consistent with experimental data shown in (Fig. 1(d)). The apparent difference between the two dispersions in Fig. 4(e) and Fig. 1(d) may be explained by (1) the well-known inadequacy of the TB model to accurately predict trigonal warping in graphene at higher energies ${ }^{41,42}$ and (2) the $3 \mathrm{NN}$ TB band structure model used here is approximated from the two dimensional experimental valence band data for pristine and gold EG along $\Gamma-\mathrm{K}-\mathrm{M}$ direction ${ }^{16}$ and a more accurate theoretical valence and conduction band dispersion along the all the high symmetry points may be required.

To further rule out the modification of electronic structure alone as a probable cause for the observed laser energy dependent Raman shift in the study, 2D Raman modes of two systems whose electronic band structure resembles pristine and gold-EG are simulated. ${ }^{43}$ Our calculations reveal that modification of the electronic structure alone results in a constant red-shift of the 2D mode of gold-EG compared to pristine EG. This clearly indicates that the excitation dependent Raman red shift in gold-EG can be understood only when modifications of both electron phonon dispersion in gold-EG are considered (supplementary material, Fig. S3).

\section{Decoupling of epitaxial graphene}

The intercalation of gold atoms between the buffer layer and monolayer graphene reduces the interaction of the top layer graphene with its substrate and locally screens the charge transfer from the substrate. It is evident from the steeper phonon dispersion in gold-EG that the no covalent interaction between gold clusters and carbon atoms in monolayer graphene and covalent bonding exists only between $\mathrm{SiC}$ and buffer layer $\mathrm{C}$ atoms. It has been known that a charge transfer between the medium surrounding graphene or hybridisation of the $\pi$-bands of graphene between adsorbed species significantly reduces the Kohn anomaly (i.e., non-analytic points in the phonon dispersion curves in the vicinity of $\mathrm{K}$ and $\Gamma$ points of the Brillouin zone, where a steeper phonon dispersion is observed due to electronphonon interactions). This reduces the slope of the spectral dispersion of the Raman 2D modes. ${ }^{44}$ However, the observed increase in the phonon dispersion slope in the current case indicates that the intercalated gold atoms favour a restoration of the band structure of EG towards an ideal graphene band structure. In the present study, the linear part of the phonon dispersion is probed using laser energies in the range of
1.5-2.5 eV, the slope of the phonon dispersion could be higher in the vicinity of the $\mathrm{K}$ point.

\section{CONCLUSION}

In conclusion, a Raman study of pristine and gold intercalated epitaxial graphene system reveals a prevailing laser excitation energy dependent red shift in the phonon modes of Gold intercalated system. In order to understand this behaviour, contributions from strain in graphene, doping, and modification of the phonon dispersion due to intercalated gold atoms are considered and finally reveal that the observed behaviour can only be explained on the basis of a modification of the phonon band structure in gold intercalated epitaxial graphene. Reduced charge transfer and interaction with the substrate favours restoration of electronic bands in graphene intercalated with gold clusters towards nearly free standing graphene and presents steeper slopes for the iTO branch along the K-M direction. The experimental data support the premise of strong modifications of the phonon band structure of gold intercalated graphene.

\section{ACKNOWLEDGMENTS}

This work as part of the European Science Foundation EUROCORES Programme DEWINT was funded under Contract No. ERAS-CT-2003-980409.

${ }^{1}$ A. C. Neto and N. M. Peres, Phys. World 19, 33 (2006).

${ }^{2}$ F. Schwierz, Nat. Nanotechnol. 5, 487 (2010).

${ }^{3}$ M. Sprinkle, M. Ruan, Y. Hu, J. Hankinson, M. Rubio-Roy, B. Zhang, X. Wu, C. Berger, and W. A. de Heer, Nat. Nanotechnol. 5, 727 (2010).

${ }^{4}$ S. Bae, H. Kim, Y. Lee, X. Xu, J.-S. Park, Y. Zheng, J. Balakrishnan, T. Lei, H. R. Kim, Y. I. Song et al., Nat. Nanotechnol. 5, 574 (2010).

${ }^{5}$ M. I. Katsnelson, K. S. Novoselov, and A. K. Geim, Nat. Phys. 2, 620 (2006).

${ }^{6}$ M. J. Word, I. Adesida, and P. R. Berger, J. Vac. Sci. Technol., B 21, L12 (2003).

${ }^{7}$ M. Yang, A. Nurbawono, C. Zhang, and Y. P. Feng, Appl. Phys. Lett. 96, 193115 (2010).

${ }^{8}$ M. Cranney, F. Vonau, P. B. Pillai, E. Denys, D. Aubel, M. M. De Souza, C. Bena, and L. Simon, Europhys. Lett. 91, 66004 (2010).

${ }^{9}$ M. Gao, Y. Pan, C. Zhang, H. Hu, R. Yang, H. Lu, J. Cai, S. Du, F. Liu, and H.-J. Gao, Appl. Phys. Lett. 96, 053109 (2010).

${ }^{10}$ M. Yankowitz, J. Xue, D. Cormode, J. D. Sanchez-Yamagishi, K. Watanabe, T. Taniguchi, P. Jarillo-Herrero, P. Jacquod, and B. J. LeRoy, Nat. Phys. 8, 382 (2012).

${ }^{11}$ Z.-G. Chen, Z. Shi, W. Yang, X. Lu, Y. Lai, H. Yan, F. Wang, G. Zhang, and Z. Li, Nat. Commun. 5, 4461 (2014).

${ }^{12}$ C.-H. Park, L. Yang, Y.-W. Son, M. L. Cohen, and S. G. Louie, Nat. Phys. 4, 213 (2008).

${ }^{13}$ B. Premlal, M. Cranney, F. Vonau, D. Aubel, D. Casterman, M. M. De Souza, and L. Simon, Appl. Phys. Lett. 94, 263115 (2009).

${ }^{14}$ K. V. Emtsev, F. Speck, Th. Seyller, L. Ley, and J. D. Riley, Phys. Rev. B 77, 155303 (2008).

${ }^{15}$ I. Gierz, T. Suzuki, R. T. Weitz, D. S. Lee, B. Krauss, C. Riedl, U. Starke, H. Höchst, J. H. Smet, C. R. Ast, and K. Kern, Phys. Rev. B 81, 235408 (2010).

${ }^{16}$ M. N. Nair, M. Cranney, F. Vonau, D. Aubel, P. L. Fèvre, A. Tejeda, F. Bertran, A. Taleb-Ibrahimi, and L. Simon, Phys. Rev. B 85, 245421 (2012).

${ }^{17}$ See www.wsi.tum.de/nextnano3 and www.nextnano.de for NEXTNANO3 device simulator.

${ }^{18}$ S. Reich, J. Maultzsch, C. Thomsen, and P. Ordejón, "Tight-binding description of graphene," Phys. Rev. B 66, 035412 (2002).

${ }^{19}$ I. Horcas, R. Fernández, J. M. Gómez-Rodríguez, J. Colchero, J. GómezHerrero, and A. M. Baro, Rev. Sci. Instrum. 78, 013705 (2007).

${ }^{20}$ C. Thomsen and S. Reich, Phys. Rev. Lett. 85, 5214 (2000). 
${ }^{21}$ R. Saito, A. Jorio, A. G. Souza Filho, G. Dresselhaus, M. S. Dresselhaus, and M. A. Pimenta, Phys. Rev. Lett. 88, 027401 (2001).

${ }^{22}$ L. M. Malard, M. A. Pimenta, G. Dresselhaus, and M. S. Dresselhaus, Phys. Rep. 473, 51 (2009).

${ }^{23}$ F. Tuinstra, J. Chem. Phys. 53, 1126 (1970).

${ }^{24}$ J. Maultzsch, S. Reich, and C. Thomsen, Phys. Rev. B 70, 155403 (2004).

${ }^{25}$ R. Narula, N. Bonini, N. Marzari, and S. Reich, Phys. Rev. B 85, 115451 (2012).

${ }^{26}$ S.-M. Choi, S.-H. Jhi, and Y.-W. Son, Phys. Rev. B 81, 081407 (2010).

${ }^{27}$ M. Mohr, J. Maultzsch, and C. Thomsen, Phys. Rev. B 82, 201409 (2010).

${ }^{28}$ O. Frank, M. Mohr, J. Maultzsch, C. Thomsen, I. Riaz, R. Jalil, K. S. Novoselov, G. Tsoukleri, J. Parthenios, K. Papagelis et al., ACS Nano 5, 2231 (2011).

${ }^{29}$ M. Huang, H. Yan, T. F. Heinz, and J. Hone, Nano Lett. 10, 4074 (2010).

${ }^{30}$ D. Yoon, Y.-W. Son, and H. Cheong, Phys. Rev. Lett. 106, 155502 (2011).

${ }^{31}$ D. Yoon, H. Moon, Y.-W. Son, B. H. Park, J. B. Kim, Y. Lee, and H. Cheong, Nano Lett. 8, 4270 (2008).

${ }^{32}$ T. Mohiuddin, A. Lombardo, R. Nair, A. Bonetti, G. Savini, R. Jalil, N. Bonini, D. Basko, C. Galiotis, and N. Marzari, Phys. Rev. B 79, 205433 (2009).

${ }^{33}$ D. M. Basko, Phys. Rev. B 78, 125418 (2008).
${ }^{34}$ S. Berciaud, X. Li, H. Htoon, L. E. Brus, S. K. Doorn, and T. F. Heinz, Nano Lett. 13, 3517 (2013).

${ }^{35}$ J. E. Lee, G. Ahn, J. Shim, Y. S. Lee, and S. Ryu, Nat. Commun. 3, 1024 (2012).

${ }^{36}$ C. Attaccalite, L. Wirtz, M. Lazzeri, F. Mauri, and A. Rubio, Nano Lett. 10, 1172 (2010).

${ }^{37}$ A. Das, S. Pisana, B. Chakraborty, S. Piscanec, S. K. Saha, U. V. Waghmare, K. S. Novoselov, H. R. Krishnamurthy, A. K. Geim, A. C. Ferrari et al., Nat. Nanotechnol. 3, 210 (2008).

${ }^{38}$ P. Venezuela, M. Lazzeri, and F. Mauri, Phys. Rev. B 84, 035433 (2011).

${ }^{39}$ P. Poncharal, A. Ayari, T. Michel, and J.-L. Sauvajol, Phys. Rev. B 78, 113407 (2008).

${ }^{40}$ F. de Juan and H. Fertig, Phys. Rev. B 85, 085441 (2012).

${ }^{41}$ A. Grüneis, J. Serrano, A. Bosak, M. Lazzeri, S. L. Molodtsov, L. Wirtz, C. Attaccalite, M. Krisch, A. Rubio, F. Mauri, and T. Pichler, Phys. Rev. B 80, 085423 (2009).

${ }^{42}$ A. Grüneis, C. Attaccalite, A. Rubio, D. V. Vyalikh, S. L. Molodtsov, J. Fink, R. Follath, W. Eberhardt, B. Büchner, and T. Pichler, Phys. Rev. B 79, 205106 (2009).

${ }^{43}$ See supplementary material at http://dx.doi.org/10.1063/1.4920931 for the simulated Raman spectrum by considering only the modification in the electronic structure of graphene.

${ }^{44}$ A. Allard and L. Wirtz, Nano Lett. 10, 4335 (2010). 